\title{
Análise da força muscular de membros inferiores em idosos: uma revisão sistemática
}

\section{Analysis of muscle strength of inferior members in elderly: a systematic review}

Análisis de la fuerza muscular de miembros inferiores en ancianos: una revisión sistemática

Viviane Eloisa Bini ${ }^{1}$

Nei Adão Ribeiro de Freitas ${ }^{2}$ Luis Paulo Gomes Mascarenhas ${ }^{3}$

${ }^{1}$ Fisioterapeuta. Mestranda do Programa de Pós-Graduação em Desenvolvimento Comunitário da Universidade Estadual do Centro-Oeste (UNICENTRO). E-mail: vivianeebini@hotmail.com, Orcid: http://orcid.org/0000-0002-9391-8317

2 Fisioterapeuta. Mestrando do Programa de Pós-Graduação em Desenvolvimento Comunitário da Universidade Estadual do Centro-Oeste (UNICENTRO). E-mail: nei_rfreitas@hotmail.com, Orcid: http://orcid.org/0000-0002-3735-6268

${ }^{3}$ Doutor em Saúde da Criança e do Adolescente, área de concentração em Endocrinologia Pediátrica pela Universidade Federal do Paraná (UFPR). Professor do Programa de Pós-Graduação Interdisciplinar em Desenvolvimento Comunitário da Universidade Estadual do Centro-Oeste (UNICENTRO). E-mail: Imascarenhas@unicentro.br, Orcid: http://orcid.org/0000-0002-7762-2727 
Resumo: A capacidade de gerar força muscular nos membros inferiores (MMIls) é um dos preditores mais importantes do status funcional em idosos e apresenta relação direta com quedas e fraturas. O salto vertical com contramovimento - Countermovement Jump (CMJ) - é uma habilidade motora fundamental na realização de inúmeras atividades, uma vez que demanda um desempenho coordenado do corpo com recrutamento muscular. Foram realizadas buscas nas bases de dados: PubMed, Bireme, SciELO e PEDro. As palavras-chave foram selecionadas de acordo com os Descritores em Ciência da Saúde (DeCS), para abordar o uso do CMJ em idosos. Dos 39 estudos encontrados, 11 foram selecionados e avaliados pela escala PEDro e apresentaram boa qualidade metodológica. O salto vertical com contramovimento se mostrou proveitoso na avaliação de força e potência em idosos.

Palavras-chave: envelhecimento; força muscular; salto vertical.

\begin{abstract}
The ability to generate muscle strength in the lower limbs (LLLS) is one of the most important predictors of functional status in the elderly and is directly related to falls and fractures. The vertical countermovement jump (CMJ) is a fundamental motor skill in the accomplishment of many activities, since it demands a coordinated performance of the body with muscular recruitment. We searched the databases: PubMed, Bireme, SciELo and PEDro. The keywords were selected according to the Health Sciences Descriptors (DeCS) to address the use of CMJ in the elderly. From 39 studies found, 11 were selected and evaluated by the PEDro scale and presented good methodological quality. The vertical countermovement jump proved to be useful in the evaluation of strength and power in the elderly.
\end{abstract}

Keywords: aging; muscle strength; vertical jump.

Resumen: La capacidad de generar fuerza muscular en los miembros inferiores (MMlls) es uno de los predictores más importantes del status funcional en ancianos y presenta relación directa con caídas y fracturas. El salto vertical con contramovimiento - Countermovement Jump (CMJ) - es una habilidad motora fundamental en la realización de innumerables actividades, una vez que demanda un desempeño coordinado del cuerpo con reclutamiento muscular. Se realizaron búsquedas en las bases de datos: PubMed, Bireme, SciELO y PEDro. Las palabras clave fueron seleccionadas de acuerdo con los Descriptores en Ciencia de la Salud (DeCS), para abordar el uso del CMJ en ancianos. De los 39 estudios encontrados, 11 fueron seleccionados y evaluados por la escala PEDro y presentaron buena calidad metodológica. El salto vertical con contramovimiento se mostró provechoso en la evaluación de fuerza y potencia en ancianos.

Palabras clave: envejecimiento; fuerza muscular; salto vertical. 


\section{INTRODUÇÃO}

O envelhecimento é um processo progressivo que acarreta perda estrutural e funcional no organismo, como declínio da capacidade funcional, perda da massa e da força muscular, resultante em grande parte da sarcopenia, perda da massa óssea e alteração hormonal, lentidão no tempo de reação, entre outros, e todos esses fatores levam à perda de autonomia e aumentam riscos de queda (HONG et al., 2015; CEDERHOLM; CRUZ-JENTOFT; MAGGI, 2013).

A capacidade de gerar força muscular rapidamente é um dos preditores mais fortes de status funcional em idosos, sendo que a redução da força muscular, potência e desempenho relacionados com a idade têm um forte impacto na função física em idosos e a redução dessas funções é especialmente importante para atividades como caminhar, subir escadas ou levantar-se de uma cadeira. Geralmente, a força muscular dos membros inferiores (MMIIs) que manifesta relação direta com quedas e fraturas é medida em grande parte apenas em pesquisas com dinamômetros ou outros equipamentos em laboratórios (BUEHRING; KRUEGER; BINKLEY, 2010; ARAUJO et al., 2015).

Existem inúmeras técnicas para avaliar funcionalidade em idosos, como Time Get Up and Go (TUG), teste de sentar e levantar da cadeira (Sit-to-Stand), teste de caminhada de seis minutos, entre outros, que são muito utilizados e considerados tradicionais (CRUZ-JENTOFT et al., 2010; MIJNARENDS et al., 2013).

Similarmente, o salto vertical com contramovimento, Countermovement Jump (CMJ), é uma habilidade motora fundamental na realização de inúmeras atividades, uma vez que demanda um desempenho coordenado do corpo com recrutamento muscular, realizando o ciclo muscular de alongamento-encurtamento (CMAE) em um curto período de tempo, e pode ser utilizado em ambientes não laboratoriais. O CMJ vem sendo utilizado no âmbito esportivo, bem como em crianças e adolescentes, para analisar funcionalidade e força, no entanto esse método não está bem elucidado em idosos (BUEHRING; KRUEGER; BINKLEY, 2010; ARAUJO et al., 2015). 
Estudos sugerem que mudanças substanciais relacionadas à idade podem ser observadas na força muscular dos membros inferiores, avaliada pelo CMJ. Estas podem ser notadas mais cedo do que deficits na força de membros superiores (MMSSs) observadas pela preensão manual ou medidas funcionais, sugerindo que o CMJ pode representar uma ferramenta importante e possivelmente clínica para detectar deficits precoces em idosos (WARD et al., 2010; BUSCHE et al., 2013).

Apesar da ampla utilização dos testes tradicionais, há perspectivas de que o CMJ poderá ser uma alternativa mais completa para a avaliação funcional em idosos, considerando que todas as variáveis que constituem as atividades de vida diária, por exemplo, tenham relação com esse teste (SHIN; DEMURA, 2009).

Diante da problemática apresentada e considerando a importância de avaliações que detectem de forma completa e mais precoce as alterações relacionadas à alteração de funcionalidade no envelhecimento, o objetivo dessa revisão sistemática é investigar as evidências científicas sobre o uso do CMJ em indivíduos idosos, fundamentando sua aplicação nessa população.

\section{MÉTODOS}

Para a elaboração desta revisão sistemática, uma busca por publicações científicas indexadas nas bases de dados PubMed, Bireme, PEDro e SciELO foi realizada no período de setembro a outubro de 2018. Foram utilizados os seguintes descritores na língua inglesa: "Jump", "Muscle Strength" e Older Adults". Dois autores, de forma independente, identificaram e selecionaram os estudos, respeitando os critérios de inclusão e exclusão estabelecidos em caso de incongruência entre ambos; o terceiro autor executava o processo de seleção.

Critérios de inclusão: (1) publicação no formato de artigo científico de estudos clínicos; (2) estudos que utilizassem o salto vertical com contramovimento como avaliação funcional de força e potência muscular; (3) publicação nos últimos cinco anos sem restrição de idioma. Foram excluídos: (1) as revisões bibliográficas, estudos de caso e estudos-piloto; 
(2) estudos que não utilizaram idosos acima de 60 anos como público-alvo; (3) estudos que não demostrassem o resultado do salto vertical com contramovimento.

Os dados foram extraídos de forma independente e incluíram informações sobre as características das publicações (título, ano de publicação), características da população (número de participantes, gênero, idade), protocolos, principais resultados e conclusões.

Nesse aspecto, a Escala PEDro foi utilizada para avaliar a qualidade metodológica e descrição estatística dos estudos. Os seguintes critérios foram pontuados: 1) elegibilidade e origem dos participantes do estudo; 2) distribuição aleatória dos participantes do estudo; 3) alocação secreta; 4) similaridade ao ponto de partida do estudo; 5) cegamento de sujeitos; 6) cegamento de terapeutas; 7) cegamento dos avaliadores; 8) análise por intenção de tratamento; 9) análise estatística intergrupos; 10) comparação entre grupos de pelo menos um desfecho primário e 11) medidas de variabilidade e estimativa dos parâmetros de pelo menos uma variável primária. A pontuação total foi gerada através da somatória dos critérios 2 a 11, sendo atribuído um (1) ponto à presença de indicadores da qualidade da evidência apresentada e zero (0) à ausência desses indicadores. O critério 1, relativo à elegibilidade e origem dos participantes, não foi considerado na pontuação final, pois está relacionado com a validade externa do estudo (GONZALEZ, 2015).

Foi utilizado o software Mendeley, um programa de desktop e web produzido pela Elsevier para gerenciar e compartilhar documentos de pesquisa, a fim de evitar a duplicata dos artigos selecionados.

\section{RESULTADOS}

Um total de 39 estudos foram identificados e, após a remoção dos duplicados (10 artigos), 29 passaram pela triagem preliminar dos títulos e resumos; foram selecionados 21 estudos potencialmente capazes de responder à pergunta desta revisão. Depois da leitura completa, 11 estudos foram incluídos. Os detalhes do processo de seleção e exclusão dos estudos estão apresentados na figura 1. 
Figura 1- Fluxograma de seleção dos estudos

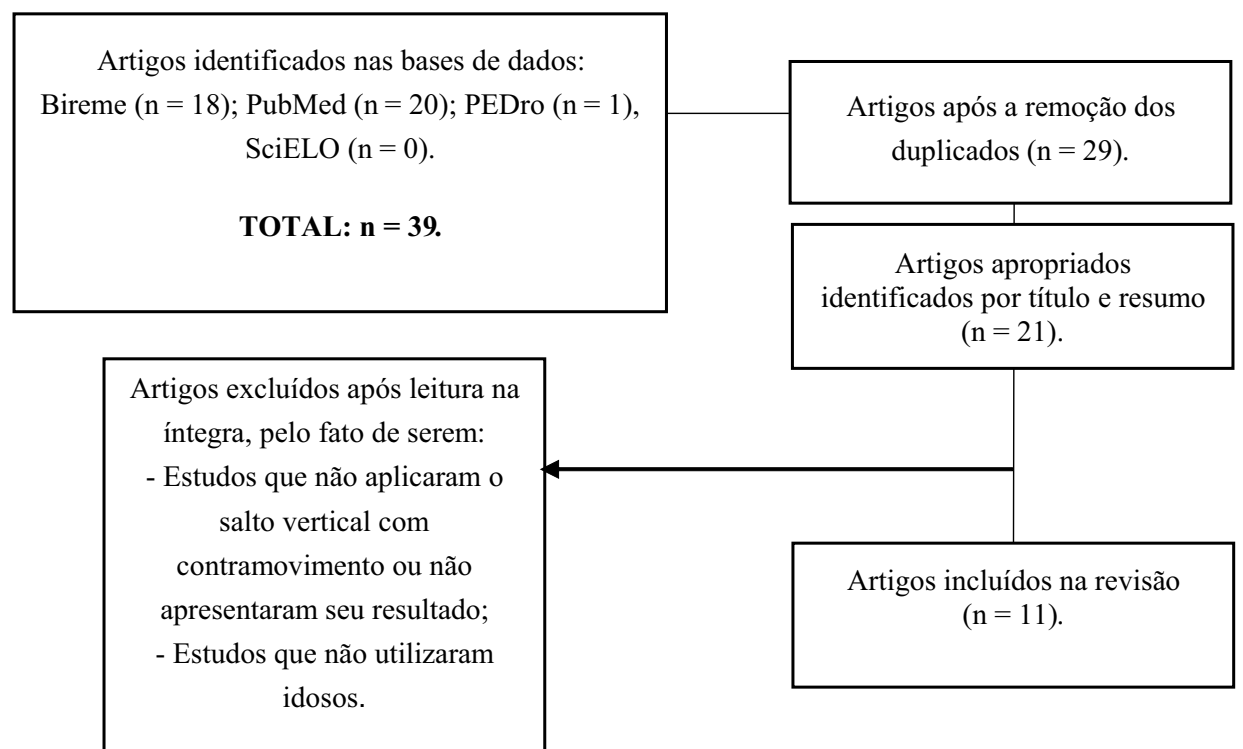

Fonte: Os autores (2018).

Todos os estudos selecionados passaram pela avaliação de qualidade metodológica PEDro, cada critério recebeu uma avaliação 0-10 (Tabela 1). A maioria dos estudos apresentou boa qualidade metodológica, oito obtiveram escore $\geq 4$ pontos e apenas um artigo apresentou escore $<4$ pontos. Apenas três artigos apresentaram alocação aleatória, quatro estudos continham similaridade entre os grupos, nenhum artigo foi positivo para cegamento dos terapeutas e dos sujeitos e um apresentou cegamento do avaliador. 


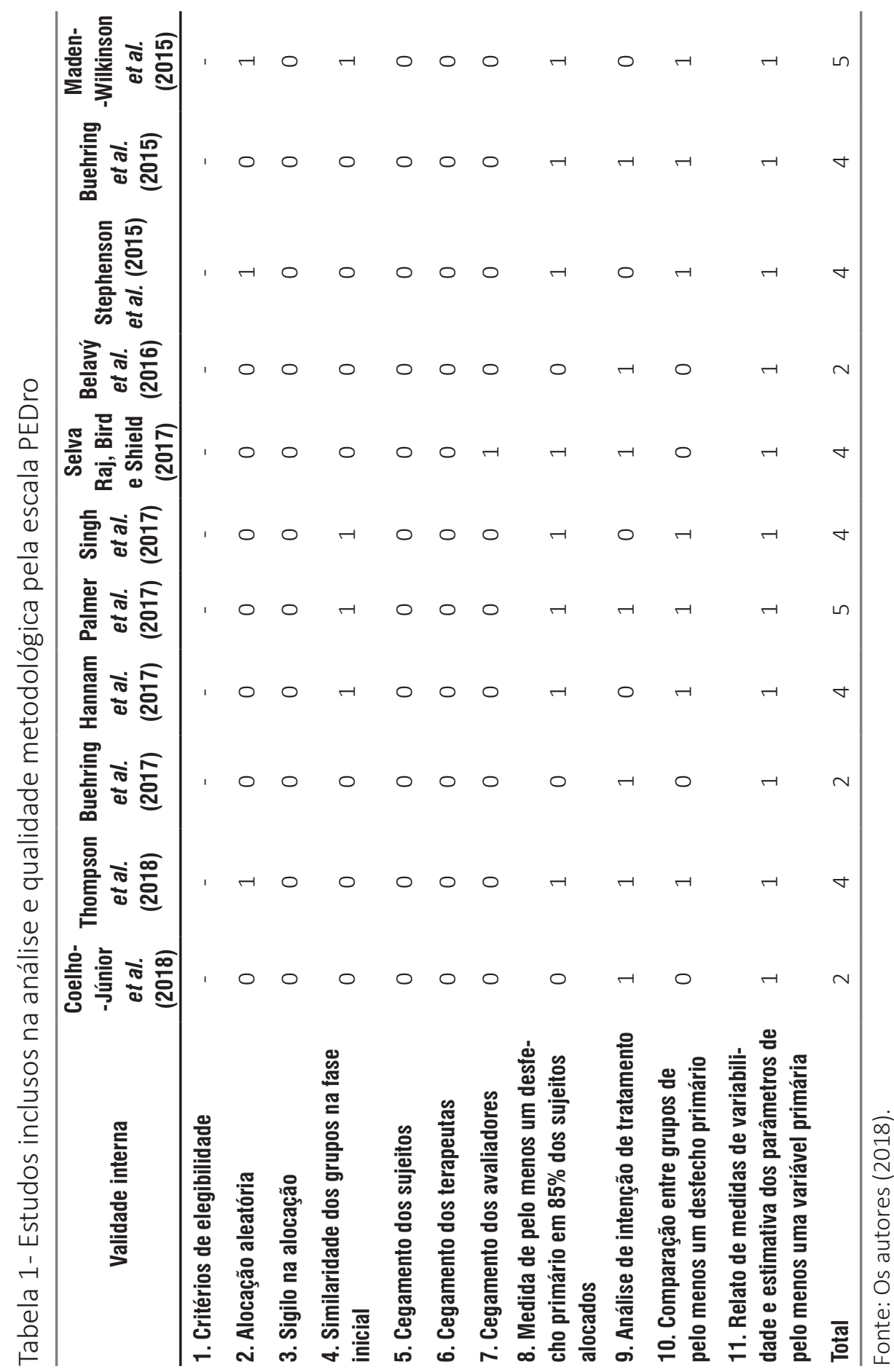


Os sujeitos participantes foram de ambos os gêneros. Todos os estudos incluíram indivíduos idosos com a idade variando de 60 a 95 anos. Houve uma variabilidade de testes utilizados juntamente do salto vertical com contramovimento (CMJ) (Tabela 2). 


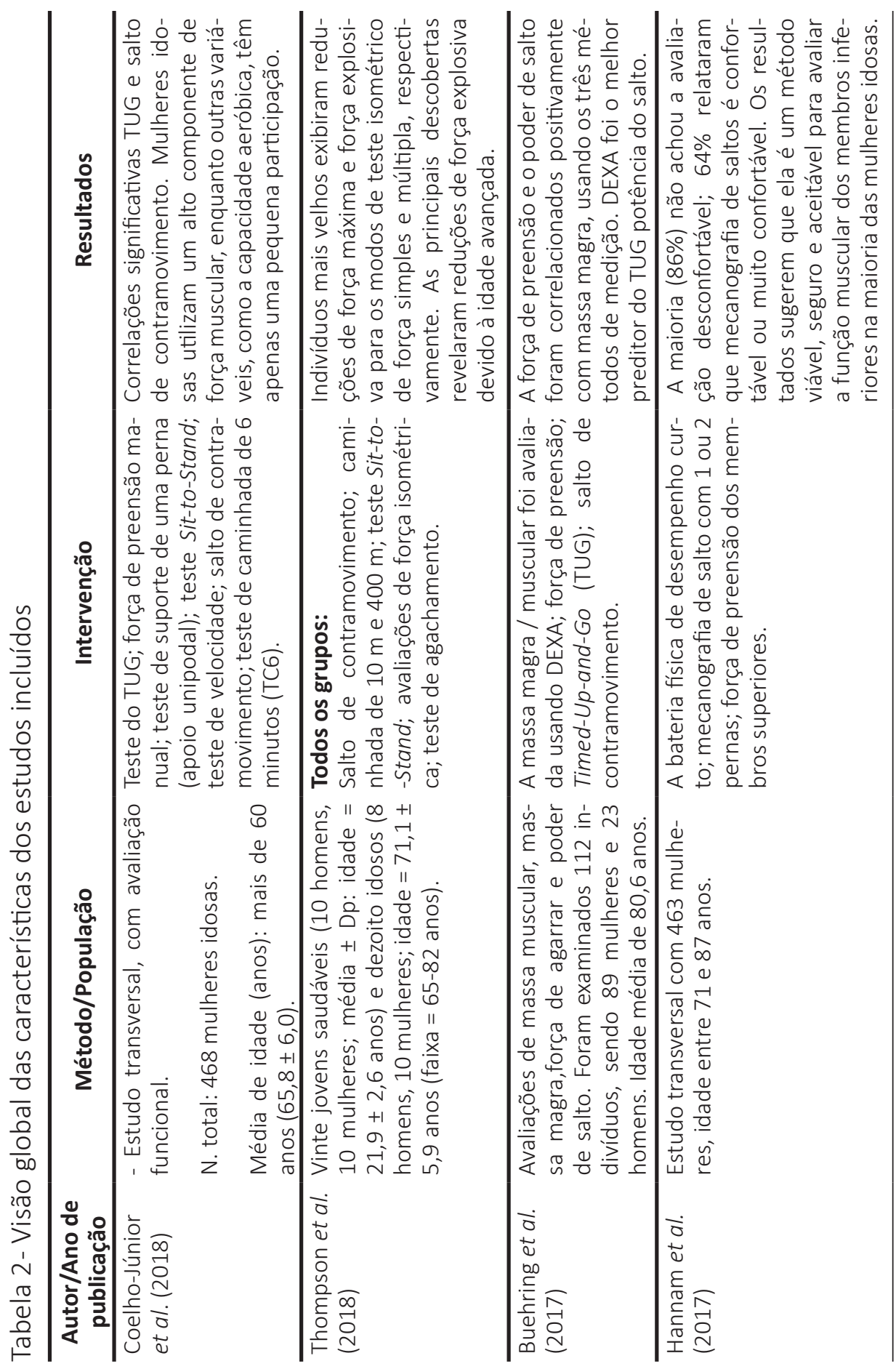




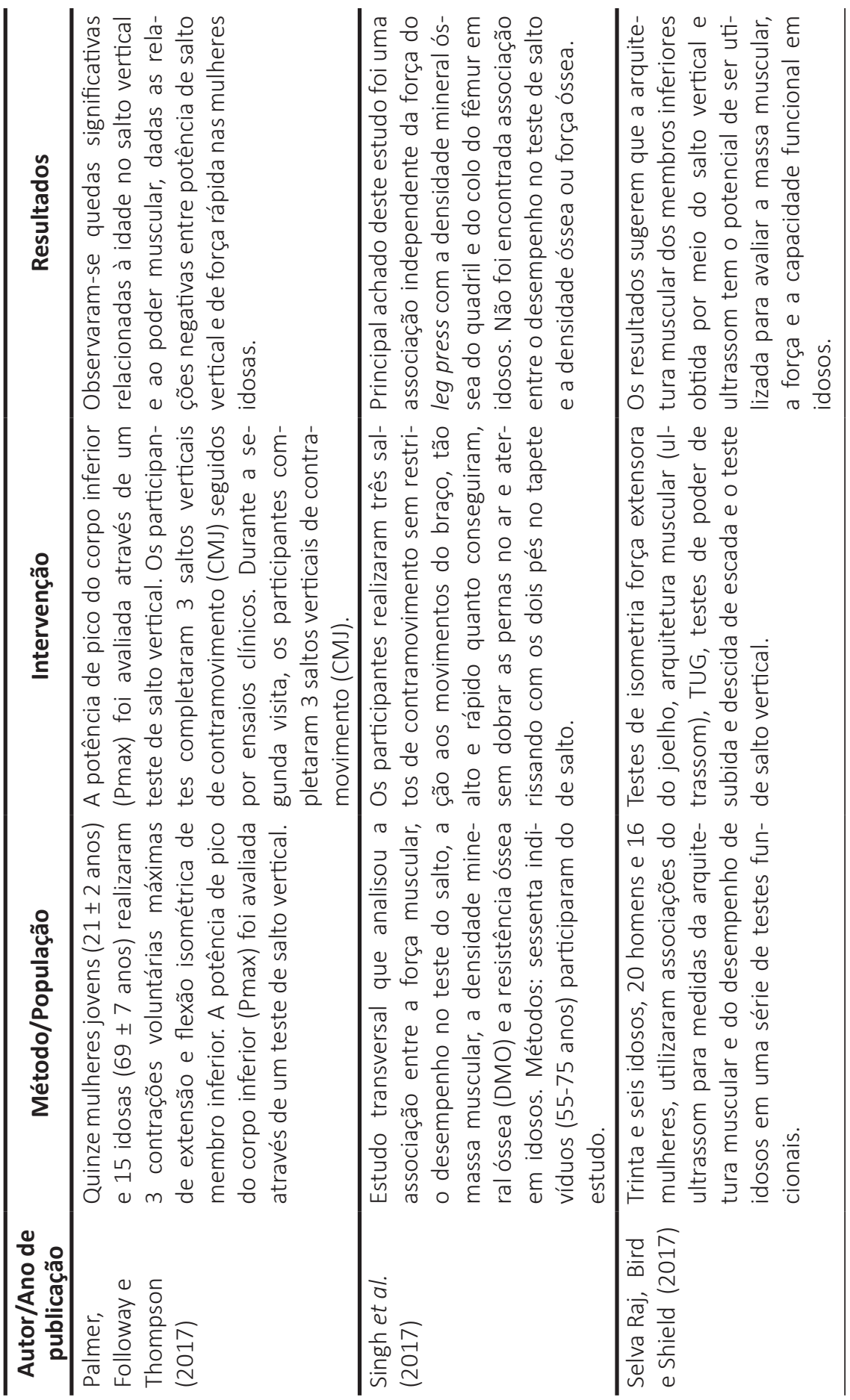




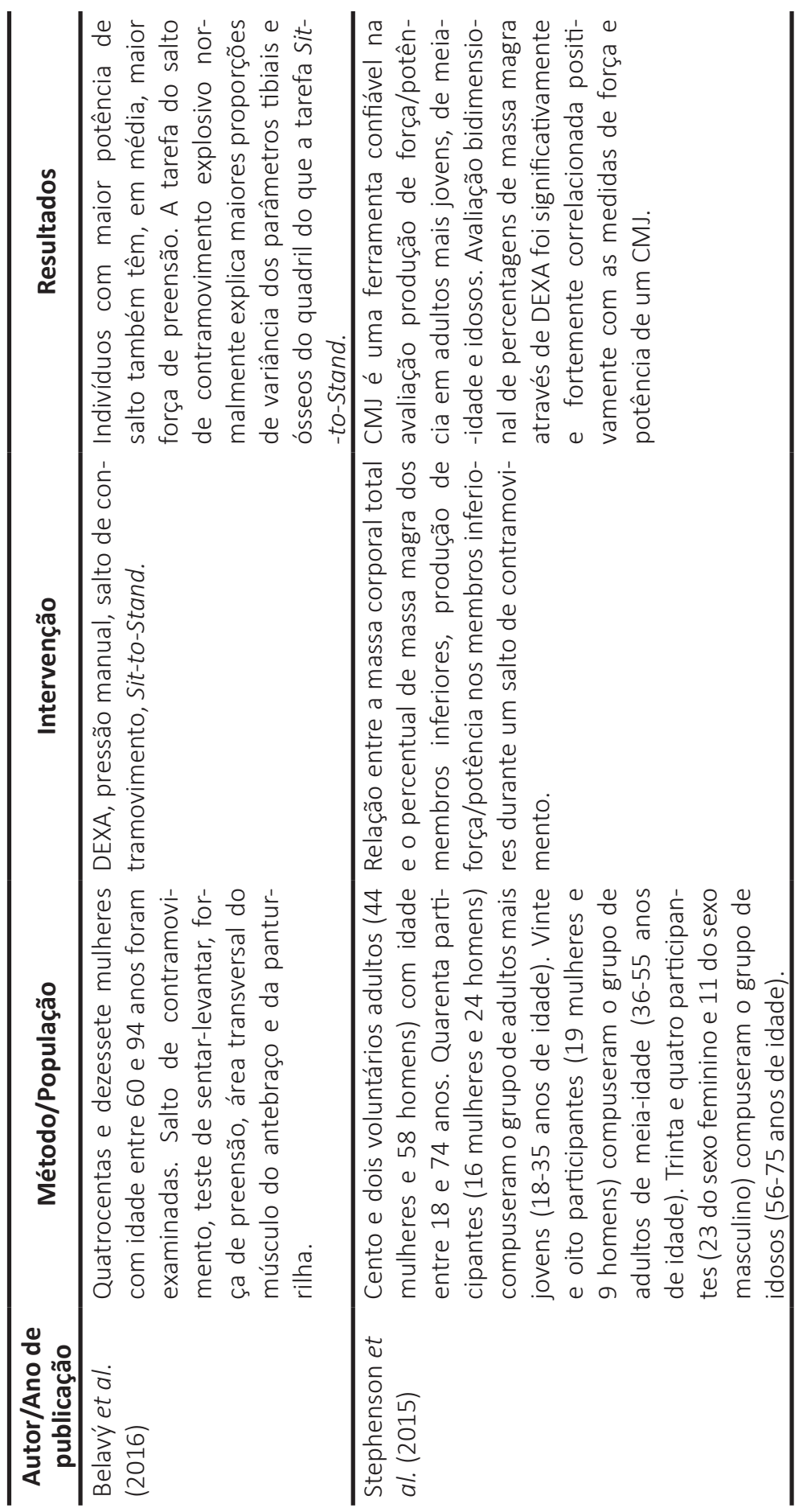




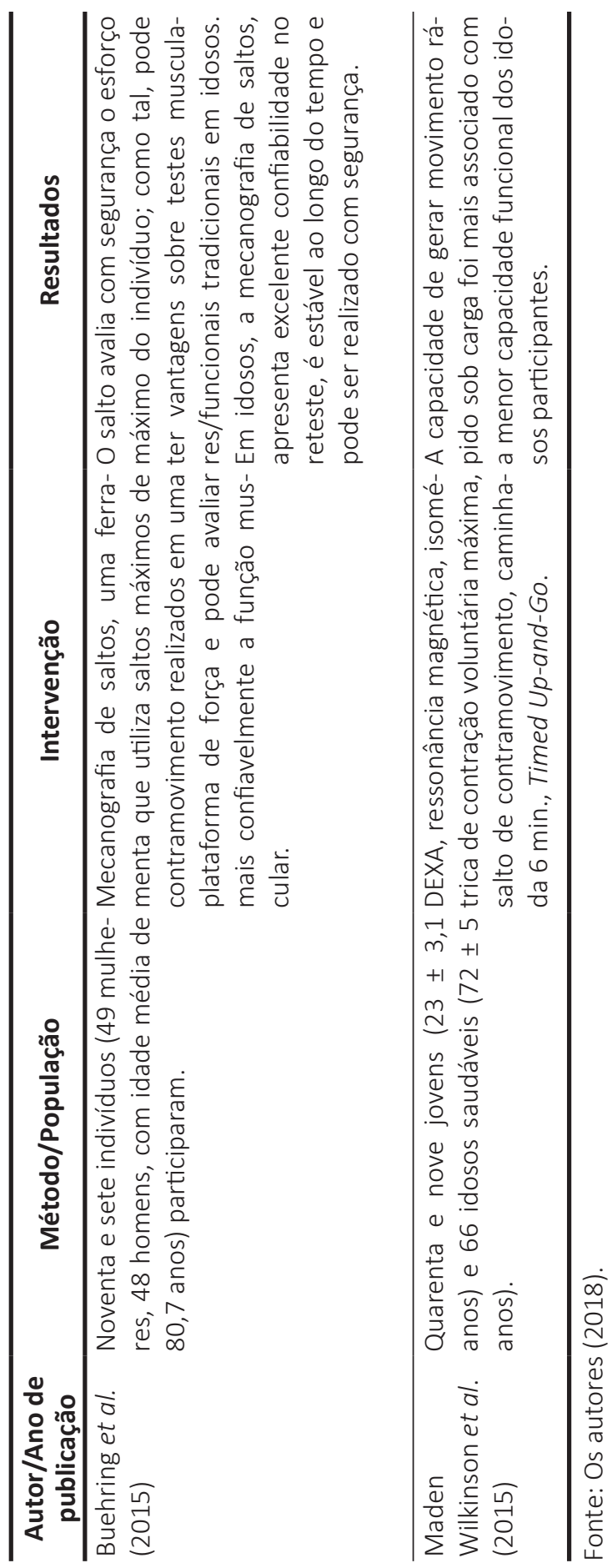




\section{DISCUSSÃO}

O presente estudo teve como objetivo investigar as evidências científicas sobre o uso do CMJ em indivíduos idosos, fundamentando sua aplicação nessa população. Houve uma variação bastante grande dos recursos que foram utilizados junto do salto para avaliar diferentes objetivos.

Dos onze trabalhos selecionados e analisados, apenas um, Singh et al. (2017), não encontrou nenhum tipo de associação significativa do CMJ com força, potência, massa muscular ou óssea; dessa forma, configura-se que mesmo protocolos diferentes podem apresentar bons resultados para detectar, principalmente, alterações musculares na população em questão.

Coelho-Júnior et al. (2018) utilizaram o salto vertical para verificar a potência dos MMlls e observaram que mulheres idosas usam um alto componente de força muscular, enquanto outras variáveis, como equilíbrio e a capacidade aeróbica, têm apenas uma pequena participação. Os resultados de correlação indicaram que o desempenho do TUG foi significativamente associado à potência do MMII; resultados da regressão linear múltipla indicaram que a maior parte da variabilidade encontrada na execução do TUG foi explicada pela força muscular dos membros inferiores.

Quatro estudos, Buehring et al. (2017), Stephenson et al. (2015), Belavý et al. (2016) e Maden-Wilkinson et al. (2015), apresentaram uma semelhança: utilizaram o método de emissão de raios $X$ de dupla energia (DEXA) como um dos comparativos com o CMJ. Eles observaram que o salto vertical foi uma boa e confiável ferramenta para investigar força e potência muscular, indicando o fato de ser uma tarefa fácil e com custos menores.

Buehring et al. (2017) identificaram correlação positiva entre testes funcionais e bioquímicos com a massa muscular magra. A potência do salto foi correlacionada positivamente com DEXA, espectroscopia de impedância bioelétrica e diluição de creatina, sendo sugerido que a mecanografia de salto tem a vantagem de medir pequenos incrementos e que estudos longitudinais adicionais devem ser realizados para examinar a possibilidade de que o CMJ seja superior na previsão de resultados relacionados à saúde.

Do mesmo modo, Stephenson et al. (2015), com objetivo de quantificar a relação entre a porcentagem de massa magra total de membros 
inferiores com DEXA, bem como a produção de força e potência do MMII durante o salto de CMJ, evidenciou que o salto é uma ferramenta confiável na avaliação de produção de força e potência, que a avaliação bidimensional de porcentagens de massa magra através de DEXA foi significativamente e fortemente correlacionada de forma positiva com as medidas de força e potência do salto.

A DEXA também foi utilizada por Belavý et al. (2016) para avaliar quais aspectos do desempenho neuromuscular estão associados à massa óssea, densidade e força. Os autores revelaram que é seguro presumir que indivíduos com maior potência de salto também tem, em média, maior força de preensão manual e que a tarefa de salto de CMJ normalmente explica maiores proporções de variância dos parâmetros ósseos tibiais e do quadril do que o teste Sit-to-Stand, por exemplo.

Em contrapartida, Singh et al. (2017), ao examinar a associação entre força muscular (leg press), desempenho no teste de salto, massa muscular, densidade mineral óssea (DMO) e força óssea, não encontraram associação entre o desempenho no teste de salto e a densidade ou força óssea; uma das limitações apontadas nesse estudo foi o tamanho da amostra.

A respeito da funcionalidade, Buehring et al. (2015), Thompson et al. (2018), Selva Raj, Bird e Shield (2017), Hannam et al. (2017) e MadenWilkinson et al. (2015), embora tenham utilizado metodologias diferentes, verificaram que o CMJ pode ser utilizado como medida funcional, sendo seguro e confiável.

Com o objetivo de examinar a confiabilidade do salto vertical CMJ em idosos de ambos os sexos, em comparação com avaliações musculares e de função física comumente usadas, Buehring et al. (2015) relataram que o salto é um teste funcional altamente reprodutível e estável ao longo do tempo, sua reprodutibilidade é semelhante ao teste de força de preensão manual, no entanto o salto tem vantagens por avaliar mais grupos musculares e ser uma atividade coordenada.

Thompson et al. (2018) apontaram em seu estudo que o desempenho de salto mostra relações muito robustas com desempenho de caminhada de curta e média distância, e Palmer, Followay e Thompson (2017), ao 
examinarem a influência da idade sobre as relações de força máxima e rápida utilizando leg press e salto vertical, notaram diminuição significativa relacionada à idade na força muscular do salto vertical.

Ao analisar as associações do ultrassom para medidas da arquitetura muscular e do desempenho de idosos em uma série de testes funcionais, em meio a eles, o CMJ, Selva Raj, Bird e Shield (2017) observaram que o maior comprimento do músculo gastrocnêmico medial está associado a um melhor desempenho no salto vertical CMJ, salientando que o salto pode ser útil para prever funcionalidade em idosos.

Para determinar a viabilidade e aceitabilidade do uso de força e potência, medidas pelo salto vertical com contramovimento, em detectar características precoces de sarcopenia em mulheres idosas, Hannam et al. (2017) utilizaram uma bateria curta de desempenho físico, observando que a força do salto está relacionada a medidas amplamente utilizadas de função muscular e mostra mudanças relacionadas a idades mais precoces.

Maden-Wilkinson et al. (2015), para investigar os motivos da redução da função física relacionada à idade, utilizaram as relações entre tamanho, força e potência muscular com teste de caminhada de 6 minutos e salto vertical em idosos e observaram que a massa muscular, determinada e analisada por DEXA, não se correlacionou com o desempenho de idosos, mas a potência, determinada a partir de um salto de contramovimento, correlacionou-se; o poder de salto foi $40 \%$ menor em idosos devido a uma menor velocidade de decolagem.

Os resultados positivos encontrados nos estudos encorajam a utilização do CMJ não apenas como ferramenta de análise de força e potência, mas também como teste funcional. A variabilidade de métodos comparativos utilizados, com bons resultados de correlação, demonstra a versatilidade do salto vertical, com a vantagem de ser um teste mais simples e de menor custo.

Limitações desta revisão sistemática incluem a possibilidade de ausência de alguns estudos devido às combinações dos termos de busca e à terminologia usada, bem como à variedade de métodos e objetivos utilizando o CMJ. 


\section{CONCLUSÃO}

De acordo com os estudos, a utilização do salto vertical com contramovimento se mostrou proveitosa na avaliação de força e potência em idosos, sendo capaz de explicar variações de massa magra e massa óssea de forma igual ou superior a testes tradicionais. Observaram-se vários aspectos de interesse com predomínio de correlação entre força, massa e funcionalidade.

Os estudos acerca do CMJ em idosos estão crescendo, mostraram-se seguros e confiáveis e é visível o intuito de sua utilização como teste para indicar funcionalidade, uma vez que ele avalia uma tarefa física que requer força e potência muscular e também requer o uso de outros sistemas que são necessários para as atividades da vida diária.

\section{REFERÊNCIAS}

ARAUJO, L. G.; ALVES, J.; MARTINS, A. C. V.; PEREIRA, G. S.; MELO, S. I. L. Salto vertical: Estado da arte e tendência dos estudos. Revista Brasileira de Ciência e Movimento, v. 21, n. 1, p. 174-181, 2015.

BELAVÝ, D. L. et al. Greater association of peak neuromuscular performance with cortical bone geometry, bone mass and bone strength than bone density: a study in 417 older women. Bone, v. 83, p. 119-26, 2016.

BUEHRING, B. et al. Comparison of muscle / lean mass measurement methods: correlation with functional and biochemical testing. Osteoporosis International, v. 29, n. 3, p. 675-83, 2017.

BUEHRING, B. et al. Reproducibility of jumping mechanography and traditional measures of physical and muscle function in older adults. Osteoporosis International, v. 26, n. 2, p. 819-25, 2015.

BUEHRING, B.; KRUEGER, D.; BINKLEY, N. Jumping mechanography: a potential tool for sarcopenia evaluation in older individuals. Journal of Clinical Densitometry, v. 13, n. 3, p. 283-91, 2010.

BUSCHE, P. et al. Mechanography in childhood: References for force and power in counter movement jumps and chair rising tests. Journal of Musculoskeletal Neuronal Interactions, v. 13, n. 2, p. 213-26, 2013. 
CEDERHOLM, T.; CRUZ-JENTOFT, A. J.; MAGGI, S. Sarcopenia and fragility fractures. European Journal of Physical and Rehabilitation Medicine, v. 49, n. 1, p. 111-7, 2013.

COELHO-JUNIOR, H. J. et al. The physical capabilities underlying timed "Up and Go" test are time-dependent in community-dwelling older women. Experimental Gerontology, v. 104, p. 138-46, 2018.

CRUZ-JENTOFT, A. J. et al. Sarcopenia: European consensus on definition and diagnosis. Age and Ageing, v. 39, n. 4, p. 412-23, 2010.

GONZALEZ, G. Z. Análise da qualidade metodológica e descrição estatística de ensaios controlados aleatorizados de intervenções fisioterapêuticas para condições musculoesqueléticas. 2015. Dissertação (Mestrado em Fisioterapia)- Universidade Cidade de São Paulo, São Paulo, 2015.

HANNAM, K. et al. Feasibility and acceptability of using jumping mechanography to detect early components of sarcopenia in community-dwelling older women. Journal of Musculoskeletal Neuronal Interactions, v. 17, n. 3, p. 246-57, 2017.

HONG, W. et al. Prevalence of sarcopenia and its relationship with sites of fragility fractures in elderly Chinese men and women. PLoS One, v. 14, n. 9, p. 352-65, 2015.

MADEN-WILKINSON, T. M. et al. Age-related loss of muscle mass, strength, and power and their association with mobility in recreationally - active older adults in the United Kingdom. Journal of Aging \& Physical Activity, v. 23, n. 3, p. 352-60, 2015.

MIJNARENDS, D. M. et al. Validity and reliability of tools to measure muscle mass, strength, and physical performance in community-dwelling older people: a systematic review. Journal of the American Medical Directors Association, v. 14, n. 3, p. 170-8, 2013.

PALMER, T. B.; FOLLOWAY, B. N.; THOMPSON, B. J. Age-related effects on maximal and rapid hamstrings/quadriceps strength capacities and vertical jump power in young and older females. Aging Clinical and Experimental Research, v. 29, n. 6, p. 1231-9, 2017.

SELVA RAJ, I.; BIRD, S. R.; SHIELD, A. J. Ultrasound measurements of skeletal muscle architecture are associated with strength and functional capacity in older adults. Ultrasound in Medicine and Biology, v. 43, n. 3, p. 586-94, 2017.

SHIN, S.; DEMURA, S. The relationship of age and leg strength in the step test with 
stipulated tempo in the elderly. Archives of Gerontology and Geriatrics, v. 49, n. 2, p. 311-6, 2009.

SINGH, H. et al. Relationship between muscle performance and DXA-derived bone parameters in community-dwelling older adults. Journal of Musculoskeletal Neuronal Interactions, v. 17, n. 2, p. 50-8, 2017.

STEPHENSON, M. L. et al. Total and lower extremity lean mass percentage positively correlates with jump performance. Journal of Strength and Conditioning Research, v. 29, n. 8, p. 2167-75, 2015.

THOMPSON, B. J. et al. Effects of age, joint angle, and test modality on strength production and functional outcomes. International Journal of Sports Medicine, v. 39, n. 2, p. 124-32, 2018.

WARD, K. A. et al. A randomized, controlled trial of vitamin D supplementation upon musculoskeletal health in postmenarchal females. Journal of Clinical Endocrinology and Metabolism, v. 95, n. 10, p. 4643-51, 2010. 